\title{
Common fixed point theorems in modified intuitionistic fuzzy metric spaces with common property (E.A.)
}

\author{
Mohammad Tanveer ${ }^{1 *}$, Mohammad Imdad ${ }^{2}$, Dhananjay Gopal ${ }^{3}$ and Deepesh Kumar Patel ${ }^{3}$
}

\author{
* Correspondence: \\ tanveer_gouri@yahoo.co.in \\ ${ }^{1}$ School of Computer \& Systems \\ Sciences, Jawaharlal Nehru \\ University, New Delhi 110 067, \\ India \\ Full list of author information is \\ available at the end of the article
}

\begin{abstract}
In this article, we utilize the notions of the property (E.A.) and common property ( $E$. A.) in the setting of modified intuitionistic fuzzy metric spaces to prove a result interrelating the property (E.A.) with common property (E.A.). Also using the common property (E.A.), we prove some common fixed point theorems in modified intuitionistic fuzzy metric spaces satisfying an implicit relation. Some related results are also derived besides furnishing an illustrative example.

AMS Subject Classification (2000): Primary 54H25; Secondary $47 \mathrm{H} 10$.

Keywords: modified intuitionistic fuzzy metric space, the property (E.A.), the common property (E.A.)
\end{abstract}

\section{Introduction and preliminaries}

The concept of fuzzy set was introduced in 1965 by Zadeh [1]. Since then, with a view to utilize this concept in topology and analysis, many authors have extensively developed the theory of fuzzy sets along with their applications (e.g., [2-9], 39). In 1986, with similar endeavor, Atanassov [10] introduced and studied the concept of intuitionistic fuzzy sets. Using the idea of intuitionistic fuzzy set, a generalization of fuzzy metric space was introduced by Park [11] which is now known as modified intuitionistic fuzzy metric space wherein notions of continuous $t$-norm and continuous $t$-conorm are employed.

Fixed point theory is one of the most fruitful and effective tools in mathematics which has enormous applications in several branches of science especially in chaos theory, game theory, theory of differential equations, etc. Intuitionistic fuzzy metric notion is also useful in modeling some physical problems wherein it is necessary to study the relationship between two probability functions as noticed in [12]. For instance, it has a concrete physical visualization in the context of two-slit experiment as the foundation of E-infinity theory of high energy physics whose details are available in El Naschie in [13-15]. Since the topology induced by intuitionistic fuzzy metric coincides with the topology induced by fuzzy metric (see [12]), Saadati et al. [16] reframed the idea of intuitionistic fuzzy metric spaces and proposed a new notion under the name of modified intuitionistic fuzzy metric spaces by introducing the idea of continuous $t$ representable.

(C) 2012 Tanveer et al; licensee Springer. This is an Open Access article distributed under the terms of the Creative Commons Attribution License (http://creativecommons.org/licenses/by/2.0), which permits unrestricted use, distribution, and reproduction in any medium, provided the original work is properly cited. 
In 1986, Jungck [17] introduced the notion of compatible mappings in metric spaces and utilized the same (as a tool) to improve commutativity conditions in common fixed point theorems. This concept has frequently been employed to prove existence theorems on common fixed points. In recent past, several authors (e.g., [18-31]) proved various fixed point theorems employing relatively more general contractive conditions. However, the study of common fixed points of non-compatible mappings is also equally interesting which was initiated by Pant [32]. Recently, Aamri and Moutawakil [33] and Liu et al. [34] respectively, defined the property (E.A.) and common property (E.A.) and utilize the same to prove common fixed point theorems in metric spaces. Most recently, Kubiaczyk and Sharma [35] defined the property (E.A.) in Menger PM spaces and utilize the same to prove results on common fixed points wherein the authors claim their results for strict contractions which are merely valid upto contractions. Similar results are also proved by Imdad et al. [23] via common property (E.A). The aim of this article is to utilize the no tion of the property (E.A.) and common property $(E . A)$ to prove some common fixed point theorems in modified intuitionistic fuzzy metric spaces. Our results generalize several previously known results in various spaces which include results in intuitionistic fuzzy metric spaces and metric spaces. Some related results are also derived besides furnishing an illustrative example.

Lemma 1.1. [36] Consider the set $L^{*}$ and operation $\leq_{L^{*}}$ defined by

$$
\begin{aligned}
& L^{*}=\left\{\left(x_{1}, x_{2}\right):\left(x_{1}, x_{2}\right) \in[0,1]^{2} \text { and } x_{1}+x_{2} \leq 1\right\} \\
& \left(x_{1}, x_{2}\right) \leq L^{*}\left(y_{1}, y_{2}\right) \Leftrightarrow x_{1} \leq y_{1} \text { and } x_{2} \geq y_{2} \text {, for every }\left(x_{1}, x_{2}\right),\left(y_{1}, y_{2}\right) \in L^{*} \text {. Then } \\
& \left(L^{*}, L_{L^{*}}\right) \text { is a complete lattice. }
\end{aligned}
$$

Definition 1.1. [10] An intuitionistic fuzzy set $\mathcal{A}_{\zeta, \eta}$ in a universe $U$ is an object $\mathcal{A}_{\zeta, \eta}=\left\{\left(\zeta_{\mathcal{A}}(u), \eta_{\mathcal{A}}(u) \mid u \in U\right)\right\}$, where, for all $u \in U, \zeta_{\mathcal{A}}(u) \in[0,1]$ and $\eta_{\mathcal{A}}(u) \in[0,1]$ are called the membership degree and the non-membership degree, respectively, of $u \in \mathcal{A}_{\zeta, \eta}$, and furthermore they satisfy $\zeta_{\mathcal{A}}(u)+\eta_{\mathcal{A}}(u) \leq 1$.

For every $z_{i}=\left(x_{i}, y_{i}\right) \in L^{*}$, if $c_{i} \in[0,1]$ such that $\sum_{j=1}^{n} c_{j}=1$ then it is easy to see that

$$
c_{1}\left(x_{1}, y_{1}\right)+\cdots+c_{n}\left(x_{n}, y_{n}\right)=\sum_{j=1}^{n} c_{j}\left(x_{j}, y_{j}\right)=\left(\sum_{j=1}^{n} c_{j} x_{j}, \sum_{j=1}^{n} c_{j} y_{j}\right) \in L^{*} .
$$

We denote its units by $0_{L^{*}}=(0,1)$ and $1_{L^{*}}=(1,0)$. Classically, a triangular norm * $=$ $T$ on $[0,1]$ is defined as an increasing, commutative, associative mapping $T:[0,1]^{2} \rightarrow$ $[0,1]$ satisfying $T(1, x)=1 * x=x$, for all $x \in[0,1]$. A triangular co-norm $S=\circ$ is defined as an increasing, commutative, associative mapping $S:[0,1]^{2} \rightarrow[0,1]$ satisfying $S(0, x)=0 \diamond x=x$, for all $x \in[0,1]$. Using the lattice $\left(L^{*}, \leq_{L^{*}}\right)$ these definitions can straightforwardly be extended.

Definition 1.2. [37] A triangular norm ( $t$-norm) on $L^{*}$ is a mapping $\mathcal{T}:\left(L^{*}\right)^{2} \rightarrow L^{*}$ satisfying the following conditions:

(I) $\left(\forall x \in L^{*}\right)\left(\mathcal{T}\left(x, 1_{L^{*}}\right)=x\right)$ (boundary condition),

(II) $\left(\forall(x, y) \in\left(L^{*}\right)^{2}\right)(\mathcal{T}(x, y)=\mathcal{T}(y, x))$ (commutativity),

(III) $\left(\forall(x, y, z,) \in\left(L^{*}\right)^{3}\right)(\mathcal{T}(x, \mathcal{T}(y, z))=\mathcal{T}(\mathcal{T}(x, y), z))$ (associativity),

(IV) $\left(\forall\left(x, x^{\prime}, y, y^{\prime}\right) \in\left(L^{*}\right)^{4}\right)\left(x \leq_{L^{*}} x^{\prime}\right)$ and $\left(y \leq_{L^{*}} y^{\prime} \rightarrow \mathcal{T}(x, y) \leq_{L^{*}} \mathcal{T}\left(x^{\prime}, y^{\prime}\right)\right)$ (monotonic-

ity). 
Definition 1.3. [36,37] A continuous $t$-norm $\mathcal{T}$ on $L^{*}$ is called continuous $t$-representable if and only if there exist a continuous $t$-norm * and a continuous $t$-conorm * on $[0,1]$ such that, for all $x=\left(x_{1}, x_{2}\right), y=\left(y_{1}, y_{2}\right) \in L^{*}$,

$$
\mathcal{T}(x, y)=\left(x_{1} * y_{1}, x_{2} \diamond y_{2}\right)
$$

Now, we define a sequence $\left\{\mathcal{T}^{n}\right\}$ recursively by $\left\{\mathcal{T}^{1}=\mathcal{T}\right\}$ and

$$
\mathcal{T}^{n}\left(x^{(1)}, \ldots, x^{(n+1)}\right)=\mathcal{T}\left(\mathcal{T}^{n-1}\left(x^{(1)}, \ldots, x^{(n)}\right), x^{(n+1)}\right)
$$

for $n \geq 2$ and $x^{(i)} \in L^{*}$.

Definition 1.4. [36,37] A negator on $L^{*}$ is any decreasing mapping $\mathcal{N}: L^{*} \rightarrow L^{*}$ satisfying $\mathcal{N}\left(0_{L^{*}}\right)=1_{L^{*}}$ and $\mathcal{N}\left(1_{L^{*}}\right)=0_{L^{*}}$. If $\mathcal{N}(\mathcal{N}(x))=x$, for all $x \in L^{*}$, then $\mathcal{N}$ is called an involutive negator. A negator on $[0,1]$ is a decreasing mapping $N:[0,1] \rightarrow$ $[0,1]$ satisfying $N(0)=1$ and $N(1)=0 . N_{s}$ denotes the standard negator on $[0,1]$ defined as (for all $x \in[0,1]) N_{s}(x)=1-x$.

Definition 1.5. [16] Let $M, N$ are fuzzy sets from $X^{2} \times(0, \infty)$ to $[0,1]$ such that $M(x, y$, $t)+N(x, y, t) \leq 1$ for all $x, y \in X$ and $t>0$. The 3-tuple $\left(X, \mathcal{M}_{M, N}, \mathcal{T}\right)$ is said to be an intuitionistic fuzzy metric space if $X$ is an arbitrary (non-empty) set, $\mathcal{T}$ is a continuous $t$ representable and $\mathcal{M}_{M, N}$ is a mapping $X^{2} \times(0, \infty) \rightarrow L^{*}$ (an intuitionistic fuzzy set, see Definition 1.1) satisfying the following conditions for every $x, y \in X$ and $t, s>0$ :

(I) $\mathcal{M}_{M, N}(x, y, t)>_{L^{*}} 0_{L^{*}}$

(II) $\mathcal{M}_{M, N}(x, y, t)=1_{L^{*}}$ if and only if $x=y$,

(III) $\mathcal{M}_{M, N}(x, y, t)=\mathcal{M}_{M, N}(y, x, t)$,

(IV) $\mathcal{M}_{M, N}(x, y, t+s) \geq_{L^{*}} \mathcal{T}\left(\mathcal{M}_{M, N}(x, z, t), \mathcal{M}_{M, N}(z, y, s)\right)$

(V) $\mathcal{M}_{M, N}(x, y,):.(0, \infty,) \rightarrow L^{*}$ is continuous.

In this case $\mathcal{M}_{M, N}$ is called an intuitionistic fuzzy metric. Here,

$$
\mathcal{M}_{M, N}(x, y, t)=(M(x, y, t), N(x, y, t)) .
$$

Remark 1.1. [38] In an intuitionistic fuzzy metric space $\left(X, \mathcal{M}_{M, N}, \mathcal{T}\right), M(x, y,$.$) is$ non-decreasing and $N(x, y,$.$) is non-increasing for all x, y \in X$. Hence $\left(X, \mathcal{M}_{M, N}, \mathcal{T}\right)$ is non-decreasing function for all $x, y \in X$.

Example 1.1. [16] Let $(X, d)$ be a metric space. Denote $\mathcal{T}(a, b)=\left(a_{1} b_{1}, \min \left\{a_{2}+b_{2}, 1\right\}\right)$ for all $a=\left(a_{1}, a_{2}\right)$ and $b=\left(b_{1}, b_{2}\right) \in L^{*}$ and let $M$ and $N$ be fuzzy sets on $X^{2} \times(0, \infty)$ defined as follows:

$$
\mathcal{M}_{M, N}(x, y, t)=(M(x, y, t), N(x, y, t))=\left(\frac{h t^{n}}{h t^{n}+m d(x, y)}, \frac{m d(x, y)}{h t^{n}+m d(x, y)}\right)
$$

for all $h, m, n, t \in R^{+}$. Then $\left(X, \mathcal{M}_{M, N}, \mathcal{T}\right)$ is an intuitionistic fuzzy metric space.

Example 1.2. [16] Let $X=\mathbb{N}$. Denote $\mathcal{T}(a, b)=\left(\max \left\{0, a_{1}+b_{1}-1\right\}, a_{2}+b_{2}-a_{2} b_{2}\right)$ for all $a=\left(a_{1}, a_{2}\right)$ and $b=\left(b_{1}, b_{2}\right) \in L^{*}$ and let $M$ and $N$ be fuzzy sets on $X^{2} \times(0, \infty)$ defined as follows:

$$
\mathcal{M}_{M, N}(x, y, t)=(M(x, y, t), N(x, y, t))=\left\{\begin{array}{l}
\left(\frac{x}{y}, \frac{y-x}{y}\right) \text { if } x \leq y \\
\left(\frac{y}{x}, \frac{x-y}{x}\right) \text { if } y \leq x
\end{array},\right.
$$


for all $x, y \in X$ and $t>0$. Then $\left(X, \mathcal{M}_{M, N}, \mathcal{T}\right)$ is an intuitionistic fuzzy metric space.

Definition 1.6. [16] Let $\left(X, \mathcal{M}_{M, N}, \mathcal{T}\right)$ be an intuitionistic fuzzy metric space. For $t>$ 0 , define the open ball $B(x, r, t)$ with center $x \in X$ and radius $0<r<1$, as

$$
B(x, r, t)=\left\{y \in X: \mathcal{M}_{M, N}(x, y, t)>_{L^{*}}\left(N_{s}(r), r\right)\right\} .
$$

A subset $A \subset X$ is called open if for each $x \in A$, there exist $t>0$ and $0<r<1$ such that $B(x, r, t) \subseteq A$. Let $\tau_{\mathcal{M}_{M, N}}$ denote the family of all open subsets of $X . \tau_{\mathcal{M}_{M, N}}$ is called the topology induced by intuitionistic fuzzy metric.

Note that this topology is Hausdorff (see Remark 3.3 and Theorem 3.5 of [11]).

Definition 1.7. [16] A sequence $\left\{x_{n}\right\}$ in an intuitionistic fuzzy metric space $\left(X, \mathcal{M}_{M, N}, \mathcal{T}\right)$ is called a Cauchy sequence if for each $0<\epsilon<1$ and $t>0$, there exists $n_{0} \in \mathbb{N}$ such that

$$
\mathcal{M}_{M, N}\left(x_{n}, y_{m}, t\right)>_{L^{*}}\left(N_{s}(\varepsilon), \varepsilon\right)
$$

and for each $n, m \geq n_{0}$ here $N_{s}$ is the standard negator. The sequence $\left\{x_{n}\right\}$ is said to be convergent to $x \in X$ in the intuitionistic fuzzy metric space $\left(X, \mathcal{M}_{M, N}, \mathcal{T}\right)$ and denoted by $x_{n} \rightarrow^{\mathcal{M}_{M, N} x}$ if $\mathcal{M}_{M, N}\left(x_{n}, x, t\right) \rightarrow 1_{L^{*}}$ whenever $n \rightarrow \infty$ for every $t>0$. An intuitionistic fuzzy metric space is said to be complete if and only if every Cauchy sequence is convergent.

Lemma 1.2. [9] Let $\mathcal{M}_{M, N}$ be an intuitionistic fuzzy metric. Then, for any $t>0, \mathcal{M}_{M, N}(x, y, t)$ is non-decreasing with respect to $t$, in $\left(L^{*}, \leq_{L^{*}}\right)$, for all $x, y \in X$.

Definition 1.8. [16] Let $\left(X, \mathcal{M}_{M, N}, \mathcal{T}\right)$ be an intuitionistic fuzzy metric space. $\mathscr{M}$ is said to be continuous on $X \times X \times(0, \infty)$ if

$$
\lim _{n \rightarrow \infty} \mathcal{M}_{M, N}\left(x_{n}, y_{n}, t_{n}\right)=\mathcal{M}_{M, N}(x, y, t),
$$

whenever a sequence $\left\{\left(x_{n}, y_{n}, t_{n}\right)\right\}$ in $X \times X \times(0, \infty)$ converges to a point $(x, y, t) \in X$ $\times X \times(0, \infty)$, i.e.,

$$
\begin{aligned}
& \lim _{n \rightarrow \infty} \mathcal{M}_{M, N}\left(x_{n}, x, t\right)=\lim _{n \rightarrow \infty} \mathcal{M}_{M, N}\left(y_{n}, y, t\right)=1_{L^{*}} \\
& \quad \text { and } \lim _{n \rightarrow \infty} \mathcal{M}_{M, N}\left(x, y, t_{n}\right)=\mathcal{M}_{M, N}(x, y, t) .
\end{aligned}
$$

Lemma 1.3. [16] Let $\left(X, \mathcal{M}_{M, N}, \mathcal{T}\right)$ be an intuitionistic fuzzy metric space. Then $\mathscr{M}$ is continuous function on $X \times X \times(0, \infty)$.

Proof. The proof is similar to that of fuzzy metric space case (see Proposition 1 of [39]).

Definition 1.9. [16] Let $f$ and $g$ be mappings from an intuitionistic fuzzy metric space $\left(X, \mathcal{M}_{M, N}, \mathcal{T}\right)$ into itself. Then the pair of these mappings is said to be weakly compatible if they commute at their coincidence point, that is, $f x=g x$ implies that $f g x$ $=g f x$.

Definition 1.10. [16] Let $f$ and $g$ be mappings from an intuitionistic fuzzy metric space $\left(X, \mathcal{M}_{M, N}, \mathcal{T}\right)$ into itself. Then the mappings are said to be compatible if

$$
\lim _{n \rightarrow \infty} \mathcal{M}_{M, N}\left(f g x_{n}, g f x_{n}, t\right)=1_{L^{*}}, \quad \forall t>0
$$

whenever $\left\{x_{n}\right\}$ is a sequence in $X$ such that

$$
\lim _{n \rightarrow \infty} f x_{n}=\lim _{n \rightarrow \infty} g x_{n}=x \in X .
$$


Definition 1.11. Let $f$ and $g$ be mappings from an intuitionistic fuzzy metric space $\left(X, \mathcal{M}_{M, N}, \mathcal{T}\right)$ into itself. Then the mappings are said to be non-compatible if there exists at least one sequence $\left\{x_{n}\right\}$ in $X$ such that $\lim _{n \rightarrow \infty} f x_{n}=\lim _{n \rightarrow \infty} g x_{n}=x \in X$ but $\lim _{n \rightarrow \infty} \mathcal{M}_{M, N}\left(f g x_{n}, g f x_{n}, t\right) \neq 1_{L^{*}}$ or non-existent for at least one $t>0$.

Proposition 1.1. [16] If self-mappings $f$ and $g$ of an intuitionistic fuzzy metric space $\left(X, \mathcal{M}_{M, N}, \mathcal{T}\right)$ are compatible, then they are weakly compatible.

The converse is not true as seen in following example.

Example 1.3. [16] Let $\left(X, \mathcal{M}_{M, N}, \mathcal{T}\right)$ be an intuitionistic fuzzy metric space, where $X$ $=[0,2]$ and $\mathcal{M}_{M, N}(x, y, t)=\left(\frac{1}{t+d(x, y)}, \frac{d(x, y)}{t+d(x, y)}\right)$ for all $t>0$ and $x, y \in X$. Denote $\mathcal{T}(a, b)=\left(a_{1} b_{1}, \min \left\{a_{2}+b_{2}, 1\right\}\right)$ for all $a=\left(a_{1}, a_{2}\right)$ and $b=\left(b_{1}, b_{2}\right) \in L^{*}$. Define selfmaps $f$ and $g$ on $X$ as follows:

$$
f(x)=\left\{\begin{array}{ll}
2 & \text { if } 0 \leq x \leq 1 \\
\frac{x}{2} & \text { if } 1<x \leq 2^{\prime}
\end{array} g(x)=\left\{\begin{array}{ll}
2 & \text { if } x=1 \\
\frac{x+3}{5} & \text { if } x \neq 1
\end{array} .\right.\right.
$$

Then we have $g 1=f 1=2$ and $g 2=f 2=1$. Also $g f 1=f g 1=1$ and $g f 2=f g 2=2$. Thus pair $(f, g)$ is weakly compatible. Again, $f x_{n}=1-\frac{1}{4 n}, \quad g x_{n}=1-\frac{1}{10 n}$. Thus $f x_{n} \rightarrow 1$, $g x_{n} \rightarrow 1$. Further $g f x_{n}=\frac{4}{5}-\frac{1}{20 n}, \quad f g x_{n}=2$. Now

$$
\begin{gathered}
\lim _{n \rightarrow \infty} \mathcal{M}_{M, N}\left(f g x_{n}, g f x_{n}, t\right)=\lim _{n \rightarrow \infty} \mathcal{M}_{M, N}\left(2, \frac{4}{5}-\frac{1}{20 n}, t\right) \\
=\left(\frac{t}{t+\frac{6}{5}}, \frac{\frac{6}{5}}{t+\frac{6}{5}}\right)<_{L^{*}} 1_{L^{*}} \forall t>0 .
\end{gathered}
$$

Hence the pair $(f, g)$ is not compatible.

Motivated by Aamri and Moutawakil [33], we have

Definition 1.12. [16] Let $f$ and $g$ be two self-mappings of an intuitionistic fuzzy metric space $\left(X, \mathcal{M}_{M, N}, \mathcal{T}\right)$. We say that $f$ and $g$ satisfy the property (E.A.) if there exists a sequence $\left\{x_{n}\right\}$ in $X$ such that

$$
\lim _{n \rightarrow \infty} \mathcal{M}_{M, N}\left(f x_{n}, u, t\right)=\lim _{n \rightarrow \infty} \mathcal{M}_{M, N}\left(g x_{n}, u, t\right)=1_{L^{*}}
$$

for some $u \in X$ and $t>0$.

Example 1.4. [16] Let $\left(X, \mathcal{M}_{M, N}, \mathcal{T}\right)$ be an intuitionistic fuzzy metric space, where $X$ $=\mathbb{R}$ and $\mathcal{M}_{M, N}(x, y, t)=\left(\frac{1}{t+|x-y|}, \frac{|x-y|}{t+|x-y|}\right)$ for every $x, y \in X$ and $t>0$. Define self-maps $f$ and $g$ on $X$ as follows:

$$
f x=2 x+1, \quad g x=x+2 .
$$

Consider the sequence $\left\{x_{n}=1+\frac{1}{n}, n=1,2, \ldots\right\}$ Thus we have

$$
\lim _{n \rightarrow \infty} \mathcal{M}_{M, N}\left(f x_{n}, 3, t\right)=\lim _{n \rightarrow \infty} \mathcal{M}_{M, N}\left(g x_{n}, 3, t\right)=1_{L^{*}}
$$

for every $t>0$. Then $f$ and $g$ satisfy the property (E.A.). 
In the next example, we show that there do exist pairs of mappings which do not share the property (E.A.).

Example 1.5. [16] Let $\left(X, \mathcal{M}_{M, N}, \mathcal{T}\right)$ be an intuitionistic fuzzy metric space, where $X$ $=\mathbb{R}$ and $\mathcal{M}_{M, N}(x, y, t)=\left(\frac{1}{t+|x-y|}, \frac{|x-y|}{t+|x-y|}\right)$ for every $x, y \in X$ and $t>0$. Define self-maps $f$ and $g$ on $X$ as $f x=x+1, g x=x+2$. In case $\exists$ a sequence $\left\{x_{n}\right\}$ such that

$$
\lim _{n \rightarrow \infty} \mathcal{M}_{M, N}\left(f x_{n}, u, t\right)=\lim _{n \rightarrow \infty} \mathcal{M}_{M, N}\left(g x_{n}, u, t\right)=1_{L^{*}}
$$

for some $u \in X$, then

$$
\lim _{n \rightarrow \infty} \mathcal{M}_{M, N}\left(f x_{n}, u, t\right)=\lim _{n \rightarrow \infty} \mathcal{M}_{M, N}\left(x_{n}+1, u, t\right)=\lim _{n \rightarrow \infty} \mathcal{M}_{M, N}\left(x_{n}, u-1, t\right)=1_{L^{*}}
$$

and

$$
\lim _{n \rightarrow \infty} \mathcal{M}_{M, N}\left(g x_{n}, u, t\right)=\lim _{n \rightarrow \infty} \mathcal{M}_{M, N}\left(x_{n}+2, u, t\right)=\lim _{n \rightarrow \infty} \mathcal{M}_{M, N}\left(x_{n}, u-2, t\right)=1_{L^{*}}
$$

so that $x_{n} \rightarrow u-1$ and $x_{n} \rightarrow u-2$ which is a contradiction. Hence $f$ and $g$ do not satisfy the property (E.A.).

Motivated by Liu et al. [34] and Imdad et al. [23,24], we also have

Definition 1.13. Two pairs $(f, S)$ and $(g, T)$ of self-mappings of an intuitionistic fuzzy metric space $\left(X, \mathcal{M}_{M, N}, \mathcal{T}\right)$ are said to satisfy the common property (E.A.) if there exist two sequences $\left\{x_{n}\right\}$ and $\left\{y_{n}\right\}$ in $X$ such that

$$
\begin{aligned}
\lim _{n \rightarrow \infty} \mathcal{M}_{M, N}\left(f x_{n}, u, t\right) & =\lim _{n \rightarrow \infty} \mathcal{M}_{M, N}\left(S x_{n}, u, t\right)=\lim _{n \rightarrow \infty} \mathcal{M}_{M, N}\left(g y_{n}, u, t\right) \\
& =\lim _{n \rightarrow \infty} \mathcal{M}_{M, N}\left(T y_{n}, u, t\right)=1_{L^{*}}
\end{aligned}
$$

for some $u \in X$ and $t>0$.

Definition 1.14. [40] Two finite families of self-mappings $\left\{f_{i}\right\}_{i=1}^{m}$ and $\left\{g_{k}\right\}_{i=1}^{n}$ of a set $X$ are said to be pairwise commuting if:

(i) $f_{i} f_{j}=f_{j} f_{i} i, j \in\{1,2, \ldots, m\}$,

(ii) $g_{k} g_{l}=g_{l} g_{k} k, l \in\{1,2, \ldots, n\}$,

(iii) $f_{i} g_{k}=g_{k} f_{i} i \in\{1,2, \ldots, m\}$ and $k \in\{1,2, \ldots, n\}$.

\section{Implicit relations}

Let $\Psi$ be the set of all continuous functions $F\left(t_{1}, t_{2}, \ldots, t_{6}\right): L^{*^{6}} \rightarrow L^{*}$, satisfying the following conditions (for all $u, v, \mathbf{1} \in L^{*}, u=\left(u_{1}, u_{2}\right), v=\left(v_{1}, v_{2}\right)$ and $1=1_{L^{*}}=(1,0)$ ):

$\left(F_{1}\right)$ : for all $u, v>_{L^{*}} 0_{L^{*}}, F(u, v, u, v, v, u) \geq_{L^{*}} 0_{L^{*}}$, or $F(u, v, v, u, u, v) \geq_{L^{*}} 0_{L^{*}}$, implies that $u \geq L^{*} v$

$\left(F_{2}\right): F(u, u, 1,1, u, u) \geq_{L^{*}} 0_{L^{*}}$ implies that $u \geq_{L^{*}} 1$.

Example 2.1. Define $F\left(t_{1}, t_{2}, t_{3}, t_{4}, t_{5}, t_{6}\right)=15 t_{1}-13 t_{2}+5 t_{3}-7 t_{4}+t_{5}-t_{6}$. Then $F \in \Psi$.

Example 2.2. Define $F\left(t_{1}, t_{2}, t_{3}, t_{4}, t_{5}, t_{6}\right)=t_{1}-\frac{1}{2} t_{2}-\frac{5}{6} t_{3}+\frac{1}{3} t_{4}+t_{5}-t_{6}$. Then $F \in \Psi$.

\section{Results}

The following lemma is proved to interrelate the property (E.A.) with common property (E.A.) in the setting of modified intuitionistic fuzzy metric spaces: 
Lemma 3.1. Let $A, B, S$ and $T$ be four self-mappings of a modified IFMS $\left(X, \mathcal{M}_{M, N}, \mathcal{T}\right)$ satisfying the following conditions:

(I) the pair $(A, S)$ (or $(B, T))$ satisfies the property (E.A.),

(II) $A(X) \subset T(X)($ or $B(X) \subset S(X)$ ),

(III) $B\left(y_{n}\right)$ converges for every sequence $y_{n}$ in $X$ whenever $T\left(y_{n}\right)$ converges (or $A\left(x_{n}\right)$ converges for every sequence $x_{n}$ in $X$ whenever $S\left(x_{n}\right)$ converges),

(IV) for all $x, y \in X, s>0, F \in \Psi$,

$$
\begin{aligned}
& F\left(\mathcal{M}_{M, N}(A x, B y, s), \mathcal{M}_{M, N}(S x, T y, s), \mathcal{M}_{M, N}(T y, B y, s),\right. \\
& \left.\quad \mathcal{M}_{M, N}(S x, A x, s), \mathcal{M}_{M, N}(A x, T y, s), \mathcal{M}_{M, N}(B y, S x, s)\right) \geq L_{L^{*}} 0_{L^{*}} .
\end{aligned}
$$

Then the pairs $(A, S)$ and $(B, T)$ share the common property (E.A.).

Proof. Since the pair $(A, S)$ enjoys the property (E.A.), there exists a sequence $\left\{x_{n}\right\}$ in $X$ such that

$$
\lim _{n \rightarrow \infty} A x_{n}=\lim _{n \rightarrow \infty} S x_{n}=z, \quad \text { for some } z \in X,
$$

implying thereby $\lim _{n \rightarrow \infty} \mathcal{M}_{M, N}\left(A x_{n}, S x_{n}, s\right)=1_{L^{*}}$. Since $A(X) \subset T(X)$, therefore for each $\left\{x_{n}\right\}$ there exists $\left\{y_{n}\right\}$ in $X$ such that $A x_{n}=T y_{n}$. Therefore, $\lim _{n \rightarrow \infty} A x_{n}=\lim _{n \rightarrow \infty} T y_{n}=z$. Thus, in all we have $A x_{n} \rightarrow z, S x_{n} \rightarrow z$ and $T y_{n} \rightarrow z$. Now, we show that $\lim _{n \rightarrow \infty} \mathcal{M}_{M, N}\left(B y_{n}, z, s\right)=1_{L^{*}}$. On using inequality (3.1), we have

$$
\begin{aligned}
& F\left(\mathcal{M}_{M, N}\left(A x_{n}, B y_{n}, s\right), \mathcal{M}_{M, N}\left(S x_{n}, T y_{n}, s\right), \mathcal{M}_{M, N}\left(T y_{n}, B y_{n}, s\right)\right. \\
& \left.\mathcal{M}_{M, N}\left(S x_{n}, A x_{n}, s\right), \mathcal{M}_{M, N}\left(A x_{n}, T y_{n}, s\right), \mathcal{M}_{M, N}\left(B y_{n}, S x_{n}, s\right)\right) \geq_{L^{*}} 0_{L^{*}}
\end{aligned}
$$

which on making $n \rightarrow \infty$, reduces to

$$
F\left(\mathcal{M}_{M, N}\left(z, \lim _{n \rightarrow \infty} B y_{n}, s\right), 1_{L^{*}}, \mathcal{M}_{M, N}\left(z, \lim _{n \rightarrow \infty} B y_{n} s\right), 1_{L^{*}}, \mathcal{M}_{M, N}\left(\lim _{n \rightarrow \infty} B y_{n}, z, s\right)\right) \geq L_{L^{*}} 0_{L^{*}} .
$$

Using $\left(F_{1}\right)$, we get $\mathcal{M}_{M, N}\left(\lim _{n \rightarrow \infty} B y_{n}, z, s\right) \geq 1_{L^{*}}$, for all $s>0$ so that $\mathcal{M}_{M, N}\left(\lim _{n \rightarrow \infty} B y_{n}, z, s\right)=1_{L^{*}}$, i.e., $\lim _{n \rightarrow \infty} B y_{n}=z$ which shows that the pairs $(A, S)$ and $(B$, T) share the common property (E.A.).

Our next result is a common fixed point theorem via the common property (E.A.).

Theorem 3.1. Let $A, B, S$ and $T$ be four self-mappings of a modified IFMS $\left(X, \mathcal{M}_{M, N}, \mathcal{T}\right)$ satisfying the condition (3.1). Suppose that

(I) the pairs $(A, S)$ and $(B, T)$ share the common property (E.A.) and

(II) $S(X)$ and $T(X)$ are closed subsets of $X$.

Then the pair $(A, S)$ as well as $(B, T)$ have a coincidence point. Moreover, $A, B, S$ and $T$ have a unique common fixed point in $X$ provided both the pairs $(A, S)$ and $(B$, $T)$ are weakly compatible.

Proof. Since the pairs $(A, S)$ and $(B, T)$ share the common property (E.A.), there exist two sequences $\left\{x_{n}\right\}$ and $\left\{y_{n}\right\}$ in $X$ such that

$$
\lim _{n \rightarrow \infty} A x_{n}=\lim _{n \rightarrow \infty} S x_{n}=\lim _{n \rightarrow \infty} B y_{n}=\lim _{n \rightarrow \infty} T y_{n}=z, \quad \text { for some } z \in X .
$$


Since $S(X)$ is a closed subset of $X$, therefore $\lim _{n \rightarrow \infty} S x_{n}=z \in S(X)$. Also, there exists a point $u \in X$ such that $S u=z$. Now, we show that $\mathcal{M}_{M, N}(A u, z, s)=1_{L^{*}}$. On using inequality (3.1), we have

$$
\begin{aligned}
& F\left(\mathcal{M}_{M, N}\left(A u, B y_{n}, s\right), \mathcal{M}_{M, N}\left(S u, T y_{n}, s\right), \mathcal{M}_{M, N}\left(T y_{n}, B y_{n}, s\right),\right. \\
& \left.\quad \mathcal{M}_{M, N}(S u, A u, s), \mathcal{M}_{M, N}\left(A u, T y_{n}, s\right), \mathcal{M}_{M, N}\left(B y_{n}, S u, s\right)\right) \geq_{L^{*}} 0_{L^{*}}
\end{aligned}
$$

which on making $n \rightarrow \infty$, reduces to

$$
F\left(\mathcal{M}_{M, N}(A u, z, s), 1_{L^{*}}, 1_{L^{*}}, \mathcal{M}_{M, N}(z, A u, s), \mathcal{M}_{M, N}(A u, z, s), 1_{L^{*}}\right) \geq_{L^{*}} 0_{L^{*}} .
$$

Using $\left(F_{1}\right)$, we get $\mathcal{M}_{M, N}(A u, z, s) \geq 1_{L^{*}}$, for all $s>0$ so that $\mathcal{M}_{M, N}(A u, z, s)=1_{L^{*},}$ that is $A u=z=S u$. Thus, $u$ is a coincidence point of the pair $(A, S)$.

Since $T(X)$ is a closed subset of $X$, therefore $\lim _{n \rightarrow \infty} T y_{n}=z \in T(X)$. Also, there exists a point $w \in X$ such that $T w=z$. Now, we show that $\mathcal{M}_{M, N}(B w, z, s)=1_{L^{*}}$ On using inequality (3.1), we have

$$
\begin{aligned}
& F\left(\mathcal{M}_{M, N}\left(A x_{n}, B w, s\right), \mathcal{M}_{M, N}\left(S x_{n}, T w, s\right), \mathcal{M}_{M, N}(T w, B w, s),\right. \\
& \left.\quad \mathcal{M}_{M, N}\left(S x_{n}, A x_{n}, s\right), \mathcal{M}_{M, N}\left(A x_{n}, T w, s\right), \mathcal{M}_{M, N}\left(B w, S x_{n}, s\right)\right) \geq \geq_{L^{*}} O_{L^{*}}
\end{aligned}
$$

which on making $n \rightarrow \infty$, reduces to

$$
F\left(\mathcal{M}_{M, N}(z, B w, s), 1_{L^{*}}, \mathcal{M}_{M, N}(z, B w, s), 1_{L^{*}}, 1_{L^{*}}, \mathcal{M}_{M, N}(B w, z, s)\right) \geq_{L^{*}} 0_{L^{*}} .
$$

Using $\left(F_{1}\right)$, we get $\mathcal{M}_{M, N}(z, B w, s) \geq 1_{L^{*}}$, for all $s>0$ so that $\mathcal{M}_{M, N}(B w, z, s)=1_{L^{*}}$, that is $B w=z=T w$. Thus, $w$ is a coincidence point of the pair $(B, T)$.

Since $A u=S u$ and the pair $(A, S)$ is weakly compatible, therefore $A z=A S u=S A u=$ $S z$. Now we need to show that $z$ is a common fixed point of the pair $(A, S)$. Now, we show that $\mathcal{M}_{M, N}(A z, z, s)=1_{L^{*}}$ On using inequality (3.1), we have

$$
\begin{aligned}
& F\left(\mathcal{M}_{M, N}(A z, B w, s), \mathcal{M}_{M, N}(S z, T w, s), \mathcal{M}_{M, N}(T w, B w, s),\right. \\
& \left.\quad \mathcal{M}_{M, N}(S z, A z, s), \mathcal{M}_{M, N}(A z, T w, s), \mathcal{M}_{M, N}(B w, S z, s)\right) \geq_{L^{*}} 0_{L^{*}}
\end{aligned}
$$

implying thereby

$$
F\left(\mathcal{M}_{M, N}(A z, z, s), \mathcal{M}_{M, N}(A z, z, s), 1_{L^{*}}, 1_{L^{*}}, \mathcal{M}_{M, N}(A z, z, s), \mathcal{M}_{M, N}(A z, z, s)\right) \geq{ }_{L^{*}} 0_{L^{*}} .
$$

Using $\left(F_{2}\right)$, we get $\mathcal{M}_{M, N}(A z, z, s) \geq 1_{L^{*}}$, for all $s>0$ so that $\mathcal{M}_{M, N}(A z, z, s)=1_{L^{*}}$, that is $A z=z$ which shows that $z$ is a common fixed point of the pair $(A, S)$.

Also $B w=T w$ and the pair $(B, T)$ is weakly compatible, therefore $B z=B T w=T B w$ $=T z$. Next, we show that $z$ is a common fixed point of the pair $(B, T)$. To accomplish this, we show that $\mathcal{M}_{M, N}(B z, z, s)=1_{L^{*}}$. On using inequality (3.1), we have

$$
\begin{aligned}
& F\left(\mathcal{M}_{M, N}(A u, B z, s), \mathcal{M}_{M, N}(S u, T z, s), \mathcal{M}_{M, N}(T z, B z, s),\right. \\
& \left.\quad \mathcal{M}_{M, N}(S u, A u, s), \mathcal{M}_{M, N}(A u, T z, s), \mathcal{M}_{M, N}(B z, S u, s)\right) \geq_{L^{*}} 0_{L^{*}}
\end{aligned}
$$

or

$$
F\left(\mathcal{M}_{M, N}(z, B z, s), \mathcal{M}_{M, N}(z, B z, s), 1_{L^{*}}, 1_{L^{*}}, \mathcal{M}_{M, N}(z, B z, s), \mathcal{M}_{M, N}(B z, z, s)\right) \geq_{L^{*}} 0_{L^{*}} .
$$

Using $\left(F_{2}\right)$, we get $\mathcal{M}_{M, N}(B z, z, s) \geq 1_{L^{*}}$, for all $s>0$ so that $\mathcal{M}_{M, N}(B z, z, s)=1_{L^{*}}$, that is $B z=z$ which showsthat $z$ is a common fixed point of the pair $(B, T)$. Uniqueness of 
the common fixed point is an easy consequence of the inequality (3.1) (in view of condition $\left.\left(F_{2}\right)\right)$.

Theorem 3.2. The conclusions of Theorem 3.1 remain true if the condition (II) of Theorem 3.1 is replaced by the following.

(II') $\overline{A(X)} \subset T(X)$ and $\overline{B(X)} \subset S(X)$.

As a corollary of Theorem 3.2, we can have the following result which is also a variant of Theorem 3.1.

Corollary 3.1. The conclusions of Theorems 3.1 and 3.2 remain true if the conditions (II) and (II') are replaced by following.

(II") $A(X)$ and $B(X)$ are closed subset of $X$ provided $A(X) \subset T(X)$ and $B(X) \subset S(X)$.

Theorem 3.3. Let $A, B, S$ and $T$ be four self-mappings of a modified IFMS $\left(X, \mathcal{M}_{M, N}, \mathcal{T}\right)$ satisfying the condition (3.1). Suppose that

(I) the pair $(A, S)$ (or $(B, T))$ satisfies the property (E.A.),

(II) $A(X) \subset T(X)($ or $B(X) \subset S(X)$ ),

(III) $B\left(y_{n}\right)$ converges for every sequence $y_{n}$ in $X$ whenever $T\left(y_{n}\right)$ converges (or $A\left(x_{n}\right)$ converges for every sequence $x_{n}$ in $X$ whenever $S\left(x_{n}\right)$ converges), and

(IV) $S(X)$ (or $T(X)$ ) be closed subset of $X$.

Then the pair $(A, S)$ as well as $(B, T)$ have a coincidence point. Moreover, $A, B, S$ and $T$ have a unique common fixed point in $X$ provided that the pairs $(A, S)$ and $(B$, T) are weakly compatible.

Proof. In view of Lemma 3.1, the pairs $(A, S)$ and $(B, T)$ share the common property (E.A.), i.e., there exist two sequences $\left\{x_{n}\right\}$ and $\left\{y_{n}\right\}$ in $X$ such that

$$
\lim _{n \rightarrow \infty} A x_{n}=\lim _{n \rightarrow \infty} S x_{n}=\lim _{n \rightarrow \infty} B y_{n}=\lim _{n \rightarrow \infty} T y_{n}=z, \quad \text { for some } z \in X .
$$

As $S(X)$ is a closed subset of $X$, on the lines of Theorem 3.1, one can show that the pair $(A, S)$ has a point of coincidence, say $u$, i.e., $A u=S u$. Since $A(X) \subset T(X)$ and $A u$ $\in T(X)$, there exists $w \in X$ such that $A u=T w$. Now, we show that $\mathcal{M}_{M, N}(B w, z, s)=1_{L^{*}}$. On using inequality (3.1), we have

$$
\begin{aligned}
& F\left(\mathcal{M}_{M, N}\left(A x_{n}, B w, s\right), \mathcal{M}_{M, N}\left(S x_{n}, T w, s\right), \mathcal{M}_{M, N}(T w, B w, s),\right. \\
& \left.\quad \mathcal{M}_{M, N}\left(S x_{n}, A x_{n}, s\right), M\left(A x_{n}, T w, s\right), \mathcal{M}_{M, N}\left(B w, S x_{n}, s\right)\right) \geq{ }_{L^{*}} 0_{L^{*}}
\end{aligned}
$$

which on making $n \rightarrow \infty$, reduces to

$$
F\left(\mathcal{M}_{M, N}(z, B w, s), 1_{L^{*}}, \mathcal{M}_{M, N}(z, B w, s), 1_{L^{*}}, 1_{L^{*}}, \mathcal{M}_{M, N}(B w, z, s)\right) \geq_{L^{*}} 0_{L^{*}} .
$$

Using $\left(F_{1}\right)$, we get $\mathcal{M}_{M, N}(z, B w, s) \geq 1_{L^{*}}$, for all $s>0$, so that $\mathcal{M}_{M, N}(B w, z, s)=1_{L^{*}}$, that is $B w=z$. Hence $B w=z=T w$. Therefore, $w$ is a coincidence point of the pair ( $B$, $T)$. The rest of the proof can be completed on the lines of Theorem 3.1.

By choosing $A, B, S$, and $T$ suitably, one can deduce corollaries for a pair as well as triod of mappings. As a simple we drive the following corollary for a pair of mappings.

Corollary 3.2. Let $A$ and $S$ be two self-mappings of a modified IFMS $\left(X, \mathcal{M}_{M, N}, \mathcal{T}\right)$ satisfying the following conditions: 
(I) the pair $(A, S)$ satisfies the property (E.A.) and $A\left(x_{n}\right)$ converges for every sequence $\left\{x_{n}\right\}$ in $X$ whenever $S\left(x_{n}\right)$ converges,

(II) $S(X)$ is closed subset of $X$ and

(III) for all $x, y \in X, s>0, F \in \Psi$,

$$
\begin{aligned}
& F\left(\mathcal{M}_{M, N}(A x, A y, s), \mathcal{M}_{M, N}(S x, S y, s), \mathcal{M}_{M, N}(S y, A y, s),\right. \\
& \left.\quad \mathcal{M}_{M, N}(S x, A x, s), \mathcal{M}_{M, N}(A x, S y, s), \mathcal{M}_{M, N}(A y, S x, s)\right) \geq \geq_{L^{*}} 0_{L^{*}} .
\end{aligned}
$$

Then the pair $(A, S)$ has a coincidence point. Moreover, $A$ and $S$ have a unique common fixed point in $X$ provided that the pair $(A, S)$ is weakly compatible.

As an application of Theorem 3.1, we can have the following result for four finite families of self-mappings. While proving this result, we utilize Definition 1.14 which is a natural extension of commutativity condition to two finite families of mappings.

Theorem 3.4. Let $\left\{A_{1}, A_{2}, \ldots, A_{m}\right\},\left\{B_{1}, B_{2}, \ldots, B_{p}\right\},\left\{S_{1}, S_{2}, \ldots, S_{n}\right\}$ and $\left\{T_{1}, T_{2}, \ldots, T_{q}\right\}$ be four finite families of self-mappings of a modified IFMS $\left(X, \mathcal{M}_{M_{1}, N}, \mathcal{T}\right)$ with $A=A_{1} A_{2} \ldots A_{m}$, $B=B_{1} B_{2} \ldots B_{p}, S=S_{1} S_{2} \ldots S_{n}$ and $T=T_{1} T_{2} \ldots T_{q}$ satisfying inequality (3.1) and the pairs $(A, S)$ and $(B, T)$ share the common property $(E . A)$. If $\mathrm{S}(\mathrm{X})$ and $\mathrm{T}(\mathrm{X})$ are closed subsets of $X$, then the pairs $(A, S)$ and $(B, T)$ have a coincidence point each.

Moreover, $A_{i}, S_{k}, B_{r}$ and $T_{t}$ have a unique common fixed point provided the pairs of families $\left(\left\{A_{i}\right\},\left\{S_{k}\right\}\right)$ and $\left(\left\{B_{r}\right\},\left\{T_{t}\right\}\right)$ commute pairwise, where $i \in\{1, \ldots, m\}, k \in\{1, \ldots, n\}$, $r \in\{1, \ldots, p\}$ and $t \in\{1, \ldots, q\}$.

Proof. The proof follows on the lines of Theorem 4.1 due to Imdad and Ali [41] and Theorem 3.1 due to Imdad et al. [40].

By setting $A_{1}=A_{2}=\ldots=A_{m}=A, B_{1}=B_{2}=\ldots=B_{p}=B, S_{1}=S_{2}=\ldots=S_{n}=S$ and $T_{1}=T_{2}=\ldots=T_{q}=T$ in Theorem 3.4, we deduce the following:

Corollary 3.3. Let $A, B, S$ and $T$ be four self-mappings of an intuitionistic fuzzy metric space $\left(X, \mathcal{M}_{M, N}, \mathcal{T}\right)$ such that the pairs $\left(A^{m}, S^{n}\right)$ and $\left(B^{p}, T^{q}\right)$ share the common property (E.A.) and also satisfy the condition (for all $x, y \in X, s>0, F \in \Psi$ ),

$$
\begin{gathered}
F\left(\mathcal{M}_{M, N}\left(A^{m} x, B^{p} y, s\right), \mathcal{M}_{M, N}\left(S^{n} x, T^{q} y, s\right), \mathcal{M}_{M, N}\left(B^{p} y, T^{q} y, s\right),\right. \\
\left.\mathcal{M}_{M, N}\left(A^{m} x, S^{n} x, s\right), \mathcal{M}_{M, N}\left(A^{m} x, T^{q} y, s\right), \mathcal{M}_{M, N}\left(S^{n} x, B^{p} y, s\right)\right) \geq \geq_{L^{*}} 0_{L^{*}}
\end{gathered}
$$

where $m, n, p$ and $q$ are positive integers. If $S^{n}(X)$ and $T^{q}(X)$ are closed subsets of $X$, then $A, B, S$ and $T$ have a unique common fixed point provided $A S=S A$ and $B T=T B$.

Finally, we conclude this article with the following example.

Example 3.1. Let $\left(X, \mathcal{M}_{M, N}, \mathcal{T}\right)$ be a modified IFMS, where $X=[0,1]$, $\mathcal{T}(a, b)=\left(a_{1} b_{1}, \min \left\{a_{2}+b_{2}, 1\right\}\right)$ for all $a=\left(a_{1}, a_{2}\right)$ and $b=\left(b_{1}, b_{2}\right) \in L^{*}$ with

$$
\mathcal{M}_{M, N}(x, y, t)=\left(\frac{t}{t+|x-y|}, \frac{|x-y|}{t+|x-y|}\right), t>0 .
$$

Define $A, B, S$ and $T$ by $A x=B x=1$,

$$
S(x)=T(x)=\left\{\begin{array}{l}
1, \text { if } x \in[0,1] \cap Q \\
\frac{1}{3}, \text { if } x \notin[0,1] \cap Q .
\end{array}\right.
$$

Also define

$$
F\left(t_{1}, t_{2}, t_{3}, t_{4}, t_{5}, t_{6}\right)=15 t_{1}-13 t_{2}+5 t_{3}-7 t_{4}+t_{5}-t_{6} .
$$


Also, $A^{2}(X)=\{1\}=S^{2}(X)$ whereas for all $x, y \in X$ and $s>0$

$$
\begin{gathered}
F\left(t_{1}, t_{2}, t_{3}, t_{4}, t_{5}, t_{6}\right)=15(1,0)-13(1,0)+5(1,0)-7(1,0)+(1,0)-(1,0) \\
=(0,0) \geq_{L^{*}} 0_{L^{*}}=(0,1)
\end{gathered}
$$

or

$$
0 \geq 0 \text { and } 0 \leq 1 \text { which is always a reality. }
$$

This demonstrates the verification of the esteemed implicit function. The remaining requirements of Corollary 3.3 can be easily verified. Notice that 1 is the unique common fixed point of $A, B, S$, and $T$.

However, this implicit function does not hold for the maps $A, B, S$, and $T$ in respect of Theorem 3.1. Otherwise, with $x=0$ and $y=\frac{1}{\sqrt{ } 2}$, we get

$$
F\left(t_{1}, t_{2}, t_{3}, t_{4}, t_{5}, t_{6}\right)=15(1,0)-13\left(\frac{t}{t+\frac{2}{3}}, \frac{\frac{2}{3}}{t+\frac{2}{3}}\right)+5\left(\frac{t}{t+\frac{2}{3}}, \frac{\frac{2}{3}}{t+\frac{2}{3}}\right)-7(1,0)\left(\frac{t}{t+\frac{2}{3}}, \frac{\frac{2}{3}}{t+\frac{2}{3}}\right)-(1,0)
$$

which contradicts the definition of $L^{*}$. Thus Corollary 3.3 is a partial generalization of Theorem 3.1 and can be situationally useful.

\section{Acknowledgements}

The authors are thankful to both the learned referees for their deep observations and pertinent suggestions which greatly helped us to improve the article significantly. We also thank to Prof. D. Mihet for some useful suggestions in the course of revision of this manuscript.

\section{Author details}

${ }^{1}$ School of Computer \& Systems Sciences, Jawaharlal Nehru University, New Delhi 110 067, India ²Department of Mathematics, Aligarh Muslim University, Aligarh 202 002, India ${ }^{3}$ Department of Applied Mathematics and Humanities, S. V. National Institute of Technology Surat, Gujarat 395 007, India

\section{Authors' contributions}

All authors carried out the proof. All authors conceived of the study and participated in its design and coordination. All authors read and approved the final manuscript.

\section{Competing interests}

The authors declare that they have no competing interests.

Received: 24 May 2011 Accepted: 6 March 2012 Published: 6 March 2012

\section{References}

1. Zadeh, LA: Fuzzy sets. Inf Control. 8, 338-353 (1965). doi:10.1016/S0019-9958(65)90241-X

2. Deng, Z: Fuzzy pseudometric spaces. J Math Anal Appl. 86, 74-95 (1982). doi:10.1016/0022-247X(82)90255-4

3. Erceg, MA: Metric spaces in fuzzy set theory. J Math Anal Appl. 69, 205-230 (1979). doi:10.1016/0022-247X(79)90189-6

4. Fang, JX: On fixed point theorems in fuzzy metric spaces. Fuzzy Sets Syst. 46, 107-113 (1992). doi:10.1016/0165-0114(92) 90271-5

5. George, A, Veeramani, P: On some results in fuzzy metric spaces. Fuzzy Sets Syst. 64, 395-399 (1994). doi:10.1016/01650114(94)90162-7

6. Grabiec, M: Fixed points in fuzzy metric spaces. Fuzzy Sets Syst. 27, 385-389 (1988). doi:10.1016/0165-0114(88)90064-4

7. Klement, EP: Operations on fuzzy sets-an axiomatic approach. Inf Sci. 27, 221-232 (1982). doi:10.1016/0020-0255(82) 90026-3

8. Kramosil, I, Michalek, J: Fuzzy metrics and statistical metric spaces. Kybernetika. 11, 336-344 (1975)

9. Saadati, R, Park, JH: On the intuitionistic topological spaces. Chaos Solitons and Fractals. 27, 331-344 (2006). doi:10.1016/j.chaos.2005.03.019

10. Atanassov, KT: Intuitionistic fuzzy sets. Fuzzy Sets Syst. 20, 87-96 (1986). doi:10.1016/S0165-0114(86)80034-3

11. Park, JH: Intuitionistic fuzzy metric spaces. Chaos Solitons and Fractals. 22, 1039-1046 (2004). doi:10.1016/j. chaos.2004.02.051

12. Gregori, V, Romaguera, S, Veereamani, P: A note on intuitionistic fuzzy metric spaces. Chaos Solitons and Fractals. 28, 902-905 (2006). doi:10.1016/j.chaos.2005.08.113

13. El Naschie, MS: A review of E-infinity theory and the mass spectrum of high energy particle physics. Chaos Solitons and Fractals. 19, 209-236 (2004). doi:10.1016/S0960-0779(03)00278-9

14. El Naschie, MS: The idealized quantum two-slit Gedanken experiment revisited-criticism and reinterpretation. Chaos Solitons and Fractals. 27, 843-849 (2006). doi:10.1016/j.chaos.2005.06.002 
15. El Naschie, MS: On two new fuzzy Kahler manifolds, Klein modular space and t Hooft holographic principles. Chaos Solitons and Fractals. 29, 876-881 (2006). doi:10.1016/j.chaos.2005.12.027

16. Saadati, R, Sedgi, S, Shobe, N: Modified intuitionistic fuzzy metric spaces and some fixed point theorems. Chaos Solitons and Fractals. 38, 36-47 (2008). doi:10.1016/j.chaos.2006.11.008

17. Jungck, G: Compatible mappings and common fixed points. Int J Math Math Sci. 9(4):771-779 (1986). doi:10.1155/ S0161171286000935

18. Aliouche, A: A common fixed point theorem for weakly compatible mappings in symmetric spaces satisfying a contractive condition of integral type. J Math Anal Appl. 322(2):796-802 (2006). doi:10.1016/j.jmaa.2005.09.068

19. Altun, I, Turkoglu, D, Rhoades, BE: Fixed Points of weakly compatible maps satisfying a general contractive conditions of integral type. Fixed Point Theory Appl 2007, 9 (2007). Article ID 17301

20. Altun, I, Turkoglu, D: Some fixed point theorems for weakly compatible mappings satisfying an implicit relation. Taiwan J Math. 13(4):1291-1304 (2009)

21. Branciari, A: A fixed point theorem for mappings satisfying a general contractive condition of integral type. Int J Math Math Sci. 29(9):531-536 (2002). doi:10.1155/S0161171202007524

22. Djoudi, A, Aliouche, A: Common fixed point theorems of Gregus type for weakly compatible mappings satisfying contractive conditions of integral type. J Math Anal Appl. 329(1):31-45 (2007). doi:10.1016/j.jmaa.2006.06.037

23. Imdad, M, Tanveer, M, Hasan, M: Some common fixed point theorems in Menger PM spaces. Fixed Point Theory Appl 2010, 14 (2010). Article ID 819269

24. Imdad, M, Ali, J, Hasan, M: Common fixed point theorems in modified intuitionistic fuzzy metric spaces. Iranian J fuzzy system. (in press)

25. Miheț, D: A generalization of a contraction principle in probabilistic metric spaces (II). Int J Math Math Sci. 5, 729-736 (2005)

26. Miheț, D: Fixed point theorems in fuzzy metric spaces using property E.A. Nonlinear Anal. 73, 2184-2188 (2010). doi:10.1016/j.na.2010.05.044

27. Rhoades, BE: Two fixed-point theorems for mappings satisfying a general contractive condition of integral type. Int J Math Math Sci. 63, 4007-4013 (2003)

28. Singh, B, Jain, S: Semicompatibility and fixed point theorems in fuzzy metric space using implicit relation. Int J Math Math Sci. 16, 2617-2629 (2005)

29. Suzuki, T: Meir-Keeler contractions of integral type are still Meir-Keeler contractions. Int J Math Math Sci 2007, 6 (2007). Article ID 39281

30. Turkoglu, D, Altun, I: A common fixed point theorem for weakly compatible mappings in symmetric spaces satisfying an implicit relation. Bol Soc Mat Mexicana. 13(3):195-205 (2007)

31. Vijayaraju, P, Sajath, ZMI: Some common fixed point theorems in fuzzy metric spaces. Int J Math Anal. 3(13-16):701-710 (2009)

32. Pant, RP: Common fixed points of noncommuting mappings. J Math Anal Appl. 188, $436-440$ (1994). doi:10.1006/ jmaa.1994.1437

33. Aamri, M, El Moutawakil, D: Some new common fixed point theorems under strict contractive conditions. J Math Anal Appl. 270, 181-188 (2002). doi:10.1016/50022-247X(02)00059-8

34. Liu, Y, Jun, Wu, Li, Z: Common fixed points of single-valued and multi-valued maps. Int J Math Math Sci. 19, 3045-3055 (2005)

35. Kubiaczyk, I, Sharma, S: Some common fixed point theorems in Menger space under strict contractive conditions. Southeast Asian Bul Math. 32, 117-124 (2008)

36. Deschrijver, G, Kerre, EE: On the relationship between some extensions of fuzzy set theory. Fuzzy Sets Syst. 133, 227-235 (2003). doi:10.1016/50165-0114(02)00127-6

37. Deschrijver, G, Cornelis, $C$, Kerre, EE: On the representation of intuitionistic fuzzy $t$-norms and $t$-conorms. IEEE Trans Fuzzy Syst. 12, 45-61 (2004). doi:10.1109/TFUZZ.2003.822678

38. Turkoglu, D, Alaca, C, Cho, YJ, Yildiz, C: Common fixed point theorems in intuition-istic fuzzy metric spaces. J Appl Math Comput. 22(1-2):411-424 (2006). doi:10.1007/BF02896489

39. Rodriguez Lopez, J, Ramaguera, S: The Hausdorff fuzzy metric on compact sets. Fuzzy Sets Syst. 147, 273-283 (2004). doi:10.1016/j.ffs. 2003.09 .007

40. Imdad, M, Ali, J, Tanveer, M: Coincidence and common fixed point theorems for nonlinear contractions in Menger PM spaces. Chaos Solitons and Fractals. 42, 3121-3129 (2009). doi:10.1016/j.chaos.2009.04.017

41. Imdad, M, Ali, J: Some common fixed point theorems in fuzzy metric spaces, Mathematical Communications. Mathematical Communications. 11, 153-163 (2006)

doi:10.1186/1687-1812-2012-36

Cite this article as: Tanveer et al:: Common fixed point theorems in modified intuitionistic fuzzy metric spaces with common property (E.A.). Fixed Point Theory and Applications 2012 2012:36. 